\title{
A review of immune amplification via ligand clustering by self-assembled liquid- crystalline DNA complexes
}

Ernest Y. Lee ${ }^{a}$, Calvin K. Lee ${ }^{a}$, Nathan W. Schmidt ${ }^{b}$, Fan Jin $^{c}$, Roberto Lande ${ }^{d, e}$, Tine Curk ${ }^{\dagger}$, Daan Frenkel ${ }^{f}$, Jure Dobnikar ${ }^{f, g, h}$, Michel Gilliet $^{d}$, and Gerard C.L. Wong ${ }^{a,{ }^{*}}$

aDepartment of Bioengineering, University of California, Los Angeles, California 90095, United States

${ }^{b}$ Department of Pharmaceutical Chemistry, University of California, San Francisco, California 94143, United States

${ }^{\mathrm{C}}$ Hefei National Laboratory for Physical Sciences at Microscale, Department of Polymer Science and Engineering, CAS Key Laboratory of Soft Matter Chemistry, University of Science and Technology of China, Hefei, P. R. China 230026

dDepartment of Dermatology, Lausanne University Hospital CHUV, 1009 Lausanne, Switzerland eDepartment of Infectious, Parasitic and Immunomediated Diseases, Istituto Superiore di Sanità, Rome, Italy

fDepartment of Chemistry, University of Cambridge, Lensfield Road CB21EW, Cambridge, UK ${ }^{9}$ Department for Theoretical Physics, Jožef Stefan Institute, Jamova 39, 1000 Ljubljana, Slovenia

hInternational Center for Soft Matter Research, Beijing University of Chemical Technology, Beijing, P. R. China 100029

*Corresponding author

\section{Abstract}

We examine how the interferon production of plasmacytoid dendritic cells is amplified by the self-assembly of liquid-crystalline antimicrobial peptide / DNA complexes. These specialized dendritic cells are important for host defense because they quickly release large quantities of type I interferons in response to infection. However, their aberrant activation is also correlated with autoimmune diseases such as psoriasis and lupus. In this review, we will describe how polyelectrolyte self-assembly and the statistical mechanics of multivalent interactions contribute to this process. In a more general compass, we provide an interesting conceptual corrective to the common notion in molecular biology of a dichotomy between specific interactions and nonspecific interactions, and show examples where one can construct exquisitely specific interactions using non-specific interactions.

Keywords: Innate immunity, TLR9, dendritic cells, interferon, liquid crystals, polyelectrolytes, like-charge attraction, statistical mechanics, multivalency, counterions, SAXS 


\section{Organization of review}

In recent work, we found that electrostatic complexes formed between anionic DNA and cationic antimicrobial peptides can greatly amplify interferon production of plasmacytoid dendritic cells ( $p D C s$ ) via Toll-Like Receptor binding ${ }^{1,2}$. This is a problem in immunology that is usually not associated with physics, but can benefit from some of the recent insights derived from polyelectrolyte physics and from the statistical mechanics of multivalent interactions. This unexpected point of contact between immunology and soft matter physics can potentially impact biomedical problems as diverse as anti-inflammatory strategies and autoimmune diseases. We first describe the immunology of Toll-Like Receptors, then summarize the work on how liquidcrystalline DNA complexes activate pDCs, and finally conclude with a review of useful biophysical concepts from multivalent binding and polyelectrolyte self-assembly. We stress that this review is not meant to be a comprehensive review of innate immunity. Rather, we try to convey in a relatively compact format the informing context important for understanding interactions between DNA and TLR9.

\section{A short introduction to Toll-like receptors in innate immunity}

Innate Immunity, Cytokines, and Type I Interferons: In the context of this review, it is helpful to tell the story backwards, starting with innate immunity. The innate immune system consists of a variety of broadly nonspecific effector cells and molecules, which include the complement system, antimicrobial peptides, neutrophils, dendritic cells, and macrophages. This is distinct from the adaptive immune system which relies on the memory and hyperspecificity of antibody-producing $B$ cells and antigen-recognizing $T$ cells. The innate immune system can sense common pathogen-associated molecular patterns (PAMPs) characteristic of infectious organisms, directly attack invading microbes, and also direct the adaptive immune system to mount a more precise response. The innate immune system can do this by inducing the secretion of the class of signaling molecules known as cytokines. Cytokines are small proteins that mediate cell-cell signaling in the immune system. Type I interferons (IFNs) are a subtype of cytokines that were initially described as important for anti-viral defense ${ }^{3}$. However, we now recognize that the function of interferons is much more general, and that other infectious agents can also induce type I IFN production. For example, type I IFNs play an important part in host defense against many bacterial infections. There are two components to type I IFN signaling the first is gene induction and release of the cytokine, and the second is binding of the cytokine to a type I IFN receptor and its activation, leading to type I IFN gene activation and downstream orchestration of immunity. (There are over 400 known interferon stimulated genes.) In addition to host defense, type I IFNs also contribute to maintenance of the haematopoietic stem cell niche, are relevant in certain cancers, play pivotal roles in many autoimmune disorders.

Innate immune receptors: The next natural question to ask is how IFNs are produced. There are multiple classes of innate receptors that sense foreign molecules and lead to production of type I IFN. These include members of the Toll-Like Receptor (TLR) family, the NOD-like receptor (NLR) family, the RIG-I-like receptor (RLR) family, and other inflammasomeassociated cytosolic receptors. Together these receptors enforce immune surveillance against various microbes. Different classes of innate immune receptors can be activated by specific microbial ligands, but generally type I IFNs are produced in response to nucleic acids (both microbial and self in origin). These nucleic acids are detected in the phagosomal or endosomal 
lumen or cytosol of innate immune cells of the myeloid lineage (macrophages, dendritic cells, and neutrophils). Once activated by microbial ligands, a family of IFN specific transcription factors known as Interferon Response Factors (IRFs) are induced ${ }^{3}$. For example, upon receptor activation IRF3 binds to the IFN- $\beta$ gene, promoting transcription and translation of the gene, and ultimately secretion of IFN- $\beta$. Secreted IFN- $\beta$ binds to and activates the type I IFN receptor present on various cells involved in immunity. This last binding event in turn leads to a series of downstream events: formation of the ISFG3 (Interferon Stimulated Factor Gene 3) complex, its translocation to the nucleus and binding to ISRE (Interferon Stimulated Response Element) sites, finally the activation of hundreds of Interferon Stimulated Genes (ISGs) ${ }^{3}$.

Toll-like receptors that induce type I IFN: In this review, we focus on the TLR family of innate immune receptors, which detects pathogens in the extracellular space or inside phagosomes or endosomes. The TLRs that induce type I IFNs include TLR2, TLR3, TLR4, TLR $7 / 8$, TLR9, and TLR13 ${ }^{4,5}$. These receptors sense viral and bacterial ligands, and in turn induce type I IFN immune responses. TLR2 and TLR4 bind to non-nucleic acid ligands to induce type I IFNs (TLR2 to bacterial lipoproteins, TLR4 to bacterial LPS (lipopolysaccharide)). Nucleic acids activate TLR3, TLR7/8, and TLR9. TLR3 binds dsRNA and can thereby function as a viral sensor. TLR7/8 binds to ssRNA, and TLR9 binds to CpG DNA.

TLR9: Of particular interest to us is the DNA-recognizing TLR9. TLR9 is expressed in multiple cell types, including plasmacytoid dendritic cells (pDCs), which are specialized dendritic cells present in the blood stream that can rapidly release large quantities of type I IFN. In pDCs, DNA within endosomes can activate TLR9, which leads to signaling via adaptor proteins MyD88 and TNF receptor associated factor 6 (TRAF6). This in turn causes large pre-made quantities of IRF7 (which is unique for this cell type) to dimerize, translocate to the nucleus, and initiate transcription of type I IFN genes ${ }^{5}$. In contrast to other innate immune cells, pDCs can quickly release large quantities of IFN- $\alpha$ because they are not dependent on induction of IFN- $\beta$ and subsequent production of IRF7 before IFN- $\alpha$ production. For this reason, pDCs are crucially important during infection, especially system infections where microbes have gained access to the blood stream. However, when employed by mistake, they can have devastating effects in the form of autoimmune diseases, such as psoriasis and lupus ${ }^{6}$.

\section{TLR9 activation by polycation-DNA complexes}

During an infection, microbial or viral DNA is taken up by pDCs into endosomes triggering IFN- $\alpha$ production via the binding of unmethylated CpG motifs to TLR9 expressed in these endosomes. Normally, host-derived self-DNA does not elicit this response because it has limited access to intracellular compartments ${ }^{7}$, but self-DNA released as a result of cell death can still bind to TLR9 receptors via its sugar-phosphate backbone ${ }^{8}$. In psoriasis, a breakdown of host segregation of self-DNA and TLR9 is hypothesized, and a pattern of aberrant IFN- $\alpha$ results. Another characteristic of psoriasis is the excessive production of antimicrobial peptides (AMPs) ${ }^{9-}$

12. Recently it was shown that self-nucleic acids can form complexes with the cationic antimicrobial peptide LL37 ${ }^{2}$. LL37 allows pDCs to recognize self-DNA through TLR9, potently activating the pDCs to over-produce type I interferons (IFN) and exacerbate the disease ${ }^{13}$. Interestingly, it has also been recently demonstrated that self-DNA complexed with cationic amyloid fibrils can activate autoimmunity. These effects may be related to the paradigm we present here, and potentially have relevance to autoimmune neurodenegerative diseases ${ }^{14}$. 
Recently, other antimicrobial peptides, including human defensins HBD2 and HBD36, and several chromatin-derived proteins, have also been found to self-assemble with DNA and promote TLR9 binding ${ }^{15}$ and activation ${ }^{16,17}$, similar to LL37. These results raised new questions about the mechanism of immune activation through TLR9. The process of complex formation can in principle confer resistance to enzymatic degradation of self-DNA by nucleases ${ }^{18}$, thus allowing TLR9 to bind to DNA inside the endosome. However, endosomal access alone does not appear to be sufficient for activation in pDCs, since many other peptides complexed with DNA are unable to activate TLR9. At this level of description, the selection criteria for TLR9 activation by DNA complexes are not well understood.

\section{Liquid-crystalline ordering of antimicrobial peptide-DNA complexes and its relation to TLR9 activation}

Since different cationic peptides (including antimicrobial peptides (AMPs)) can form electrostatic complexes with DNA, but with different outcomes in terms of pDC activation, it is sensible to solve the structures of these complexes using Small Angle X-ray Scattering (SAXS). Once these structures are known, then we will be in a position to address two questions relevant to the differential pDC activation: 1) Do these different self-assembled structures lead to different cell entry mechanisms and thereby different levels of endosomal access? 2) Do differences in self assembled structures interact differently with TLR9 receptors once endosomal access is achieved?

The relationship between $\mathrm{pDC}$ activation and the structure of DNA complexes can be roughly assessed by comparing three prototypical examples of DNA-peptide complexes. HIV TAT is a cell-penetrating peptide that can translocate across membranes ${ }^{19}$ and therefore has endosomal access. However, incubation of pDCs with TAT-DNA complexes does not produce significant levels of IFN- $\alpha$ in pDCs (Fig. 1). Furthermore, TAT-DNA complexes efficiently enter TLR9-containing endosomal compartments (Fig. 2b). The behavior of the TAT peptide suggests that endosomal access alone is not sufficient for strong IFN induction in pDCs. SAXS measurements show that DNA is organized into a columnar arrangement within TAT-DNA complexes similar to many phases of DNA (and other biological polyelectrolytes) condensed by multivalent cations ${ }^{20}$. TAT-DNA complexes form a columnar hexagonal lattice with parameters

$\mathrm{a}=2.90 \mathrm{~nm}, \mathrm{c}=3.50 \mathrm{~nm}$ (Fig. 1a,3d) ${ }^{21}$, which corresponds to a bundle-like complex with closepacked DNA (Fig. 1a) ${ }^{1}$. Human beta-defensin-3 (HBD3) is also polycationic, and is capable of condensing DNA and reaching TLR9 in endosomes (Fig. 2b). In contrast to the behavior of the TAT peptide, however, HBD3-DNA complexes induce strong $2200 \mathrm{pg} / \mathrm{ml}$ IFN- $\alpha$ production in pDCs, which is $\sim 100 x$ higher than that from TAT-DNA complexes. SAXS measurements (Fig. 1a) show that HBD3-DNA complexes also form a columnar phase. However, the structure is different: The inter-DNA spacing in HBD3-DNA complexes is $3.25 \mathrm{~nm}$, which is significantly larger than the spacing in TAT-DNA complexes. Finally, we examine LL37. LL37 can enter endosomes, access TLR9 receptors (Fig. 2b) and induce high levels of IFN- $\alpha$ production in pDCs (Fig. 1b), similar to HBD3-DNA complexes. Like HBD3-DNA complexes, LL37-DNA complexes also have a columnar structure with a relatively large inter-DNA spacing $(a=3.40$ $\mathrm{nm}$ ) (Fig. 3a,b). These measurements suggest that the inter-DNA spacing in liquid-crystalline 
columnar DNA-complexes may be an important parameter in determining the level of pDC IFNa production.

To test this basic idea, pDC activation was measured for a variety of DNA complexes with different inter-DNA spacings. The above analysis was repeated with a diverse set of natural and synthetic cationic molecules, including peptides (R9, TAT, polylysine (MW 70,000), hBD3d, NLS, penetratin, HBD3, LL37) as well as non-peptide cationic molecules (hexamine cobalt, tris(ethylenediamine) cobalt, protamine sulfate, PEI750k, PAMAM dendrimers (G3, G4, G6) $)^{1}$. These cationic molecules condense DNA into ordered columnar complexes with a range of inter-DNA spacings from $\mathrm{a}_{\text {HexamineCo }}=2 \pi / \mathrm{q}_{\text {HexamineCo }}=2.43 \mathrm{~nm}$ for Hexamine cobalt (III) to $\mathrm{a}_{\mathrm{G} 3}$ $=2 \pi / \mathrm{q}_{\mathrm{G} 3}=4.37 \mathrm{~nm}$ for PAMAM dendrimer G3 (Fig. 1b). An important criterion for pDC activation in these DNA complexes is the inter-DNA spacing. High resolution synchrotron SAXS can be used to solve the self-assembled structures of these complexes, and provide accurate measurements of the inter-DNA spacing. High levels of IFN- $\alpha$ production are induced by complexes with SAXS first peak positions between 1.8-2.0 $\mathrm{nm}^{-1}$ corresponding to inter-DNA spacings near a $~ 3.5 \mathrm{~nm}$ (Fig. 1b), whereas complexes with spacings that significantly differ from this value induce weaker responses. Changes in $\mathrm{NaCl}$ concentration levels over the range of physiological values present in endosomes can in principle change the inter-DNA spacing slightly. In our measurements, we find that monovalent salt levels did not alter the structures of representative polycation-DNA complexes or their inter-DNA spacings significantly, and certainly not to a degree sufficient to change activation profiles ${ }^{1}$. Importantly, DNA complexed with polycations that induce strong IFN production and those that induce weak IFN production can both access endosomes and both colocalize with TLR9 (Fig. 2). This microscopy result addresses the first of our questions outlined above: The colocalization of non-inducing complexes with TLR9 demonstrates that cellular entry and trafficking cannot be solely responsible for their lack of activity. The precise quantitative efficiency of endosomal access may, in principle, modulate the degree of IFN- $\alpha$ production induced by specific polycation-DNA complexes. To address the second of our questions above regarding whether different selfassembled structures can impact how these complexes interact with TLR9 receptors: The correlation of structural results with pDC activation measurements show that the presentation of spatially periodic DNA with spacing comparable to TLR receptor size can dramatically increase pDC IFN- $\alpha$ production. The optimum range of inter-DNA spacing at 3-4 $\mathrm{nm}$ spacing is quite suggestive. The low distance cut-off is roughly the steric size of TLR receptors ${ }^{22-24}$, which defines the distance of closest approach between receptors. The large distance cut-off is consistent with strong electrostatic interactions expected in this system, which allows a 'grill'-like arrangement of parallel anionic DNA to interact with both the inside and outside surfaces of adjacent cationic TLR9 receptors, and thereby 'crosslink' such receptors into a zipper-like ligand-receptor array (Fig. 1c,d).

A single columnar DNA complex ('DNA bundle') can bind transversely and effectively present a 'vicinal surface', a spatially-periodic 'grill'-like array of parallel DNA chains to multiple TLR9 receptors. Because of this, there is potential for multivalent binding effects (Fig. 1d). The degree of multivalency can be estimated via the measured domain size (Fig. 3a) of DNA ordering, extracted from the measured SAXS peak widths (Fig. 3b-e). Here it is interesting to 
consider the statistical mechanics of multivalent ligand receptor binding. Generally, in systems where ligands are able to form multiple weak bonds with target receptors, one can expect to observe 'super-selectivity' (which was originally used to described multivalent interactions for nanoparticles with decorated with DNA ${ }^{25}$. In these systems, binding increases sharply with receptor concentration ${ }^{25}$. Binding of immune complexes to TLR9 receptors is more complex, and depends sensitively on inter-DNA spacing in the self-assembled complex or DNA bundle. The data shows a dramatic $\sim 100 \mathrm{x}$ increase in IFN- $\alpha$ production as the distance between DNA chains in a complex shifts $\sim 0.5 \mathrm{~nm}$. These large systems are at present not accessible to allatom simulations, which necessarily involve LL37, DNA, and TLR9. We therefore developed a coarse-grained computational model. Details of this model are described in Schmidt et. al. ${ }^{1}$. A key part of the model is the electrostatic interactions between the anionic DNA and the cationic binding surfaces of TLR9 receptors. The coarse-grained model shows that the binding is strongest for parallel DNA chains with inter-DNA spacings that allow them to fit in with an array of closely-packed TLR9'. This arrangement allows 'interdigitation' between TLR9 and the periodic DNA array, where multiple receptors and ligands interlock in an alternating manner. Such optimal ordered structures can recruit and bind many TLR9 molecules and trigger an orders-of-magnitude stronger pDC response than mismatched structures with larger and smaller inter-DNA spacings ${ }^{1}$.

The simplified quantitative model presented in Schmidt et. al. ${ }^{1}$ strongly suggests that electrostatic self-assembly between DNA and polycations can result in a 'clustering' of immune ligands. This clustering drives a significant amplification of the number of active TLR9 receptors. It is interesting to compare these results to recent work on amplification via synapse-like receptor clustering ${ }^{26}$. In the present work, IFN production is amplified when ligands rather than receptors cluster at the right spacing to recruit and bind with TLRs. Importantly, TLR9 activation depends on both the inter-DNA spacing and the number of DNA ligands in the complex, since both factors contribute to electrostatics, multivalency, and thereby binding amplification. (We note that the above effects can operate in conjunction with other processes such as protection from enzymatic degradation, receptor cooperativity and clustering, and downstream consequences of active TLR9 receptors. For example, the addition of receptor clustering effects will further enhance the selectivity/amplification.) However, now that we have a model for this type of TLR driven pDC activation, it suggests that some of the polyelectrolyte physics developed in the last few decades, which are not usually associated with immunology literature, may be relevant to the process. Here, we will selectively review some of these effects.

\section{The statistical mechanics of multivalent interactions and superselectivity}

The adaptive immune system employs antibodies that bind to very specific motifs on infectious agents, marking them for destruction. However, molecular recognition in the innate immune system is more primitive, with non-specific recognition of a variety of ligands that have similar structural and physiochemical character. In molecular and cell biology, there is a common notion that there is a kind of dichotomy between specific and non-specific interactions. We think of the failure of molecular recognition in terms of specific interactions becoming non- 
specific. Recent work, including our work here, show examples of the opposite, where nonspecific interactions of PAMPs (such as dsDNA) with the innate immune system can actually be organized into amplified, specific interactions, with consequences for autoimmune disorders and targeted drug delivery.

Ligand-receptor signaling is a cornerstone of molecular biology. Cells and tissues communicate by sending and receiving messages in the form of proteins, small molecules, and nucleic acids. When engaging stochastic cellular phenomena like signaling, the first sensible step is to describe such interactions as a single ligand binding to a single receptor, triggering downstream activation of other signaling molecules. There are many examples where multivalent interactions are required in order to obtain a cellular response. These are interactions in which multiple ligands of one object bind to multiple receptors of another. There are many examples of this behavior in the immune system, where it is important to discern signal from noise. Multivalent antigens can cross-link multiple lgM receptors at once on the surface of B cells, leading to a T-cell independent activation of the immune system ${ }^{27}$. Multivalent antigen-receptor interactions by multivalent CD40 ligands can also trigger B cell activation and immunoglobulin synthesis ${ }^{28}$. Another example of multivalent interactions includes the binding of hyaluronic acid (HA) to CD44 receptors on the surfaces of cells, mediating cell adhesion to the extracellular matrix ${ }^{29}$. Lesley et. al. found that an increase in the oligomer size of HA led to a concomitant superlinear increase in avidity of CD44 binding. Interestingly, proliferation of the HA-rich pericellular matrix has been associated with amplification of CD44 signaling in inflammation ${ }^{30}$ and tumor formation ${ }^{31}$. Thus, the HA-CD44 interaction has become an important target for the development of anti-cancer drugs. Cancer-targeting nanoparticles coated with multiple ligands also take advantage of superselectivity by inducing multivalent interactions with rogue cells that express a higher density of a particular receptor relative to normal cells ${ }^{32}$. In the context here, we have found that a self-assembled LL37-DNA complex can cross-link multiple TLR9 receptors within the endosomes of pDCs, leading to receptor recruitment and amplification of the immune response ${ }^{1}$. These examples highlight the importance of multivalent ligand-receptor signaling in normal and abnormal physiology, and in the treatment of disease.

The statistical mechanics of multivalent ligand-receptor interactions has been recently examined experimentally, theoretically, and computationally, and it leads naturally to the concept of superselectivity. Superselectivity is defined by a superlinear (steeper than linear) relationship between the surface density of receptor sites and the surface density of bound ligands. This under-appreciated aspect of multivalent interactions allows a small change in receptor density to drive a large change in the number of bound ligands, in a manner that depends on the degree of multivalency. Using a model system of multivalent 'guest/host' interactions, in which 'host' $\beta$-cyclodextrin $(\beta-C D)$ is grafted onto a hyaluronic acid polymer backbone, and 'guest' ferrocene functionalized on a self-assembled monolayer (SAM) surface, Dubacheva et. al. discovered that the amount of bound ferrocene increased faster than linearly with increases in the density of surface binding sites ${ }^{33}$. Interestingly, receptor recruitment and clustering was also observed, which is analogous to the TLR9 work above. In a different experiment, spatiotemporal control over the monomer ordering in a supramolecular polymer 
was achieved, via the binding of ssDNA to cationic receptors in a multivalent manner. The experimental system is based on 1,3,5-benzenetricarboxamide derivatives (BTA). Supramolecular copolymers are made by assembling two BTA monomer subtypes, neutral and charged. The charged BTA monomers can interact electrostatically and act as receptors. The introduction of ssDNA resulted in the recruitment and binding of receptors within the assembled copolymer. FRET results showed that exposure of sSDNA triggered clustering of the cationic subtypes of BTA receptors via reorganization of the polymer, forming "islands" of similar receptors in a superselective fashion ${ }^{34}$. In both cases, these model systems are validated using statistical mechanical models. For example, these models predict that a relatively low receptorligand binding affinity and a threshold receptor concentration are required for superselectivity. Above the threshold concentration, ligands saturate the receptors very quickly despite each individual interaction being weak. Below the threshold concentration, however, ligands do not bind appreciably to the targets ${ }^{25}$. With this perspective, one can see that what is often called binding specificity (greatly increased binding of one specific component) and binding amplification are in fact related concepts. Superselectivity, a downstream consequence of multivalent interactions, can increase binding specificity, receptor recruitment, and subsequent amplification of downstream responses.

Assuming the size and the shape of the bundles as observed in the experiments, it is possible to predict the amplification of the receptor activation based on a simple coarse-grained theory and statistical mechanics of multivalent binding. The model contains steric and electrostatic interactions between TLR9 receptors and DNA bound in a bundle of a fixed size (a crystallite with 6x6 DNA helices of length $20 \mathrm{~nm}$ ) but with variable DNA-DNA spacing a. As measured in the experiments, we assume that the electrostatic attraction between a negatively charged single DNA molecule and a TLR9 receptor is not sufficiently strong to result in appreciable binding affinity $\varepsilon$. The core assumption of the model is that the electrostatic interactions are amplified by the backbone attraction between the TLR9 receptor and DNA molecules arranged in a grill-like pattern. The effective binding affinity for a bundle interacting with a receptor is then $\varepsilon^{*}=\varepsilon+2 U(a)$, where the backbone attraction contribution $U(a)$ is

$U(a)=-B \frac{e^{-\kappa(a-\sigma / 3)}}{(a-\sigma / 3)}+\left(\frac{\sigma}{12(a-2 \sigma / 3)}\right)^{12}$.

Here $\sigma$ is the typical width of the TLR9 receptor, $B \sim 20 k_{B}$ T (see SI of [1]) is the magnitude of the electrostatic interaction and $\kappa^{-1}=0.8 \mathrm{~nm}$ is the Debye screening length at physiological conditions. The backbone attraction depends on the lattice spacing $a$ : at large $a$, it is negligible, but it increases sharply when the spacing becomes of the order of $\sigma$. At very small DNA separations, due to the steric effects, it decreases again. Through our model, the binding affinity -- and with it the expected number of bound receptors $n_{B}$ to one DNA bundle - clearly depend on the lattice spacing. A bundle is considered bound to the cell, if there is at least one receptor bound to it. The expected number of bound bundles is related to the free energy $F$ of bundlereceptor binding, $\left\langle N_{D N A}\right\rangle \propto e^{-F / k_{B} T}$, and the total number of active receptors, $N_{\mathrm{A}}$, is

$$
N_{A}(a)=n_{B}(a)\left\langle N_{D N A}\right\rangle \mu n_{B}(a) e^{F(a) / k_{B} T}
$$


The free energy $F$ can be obtained from computer simulations and also estimated by a simple Langmuir adsorption theory. It has been shown in [1] that the number of bound receptors to a bundle exponentially depends on the binding affinity $\varepsilon^{*}$ and through it on the spacing $a$, while the total activation $N_{\mathrm{A}}$ exhibits a much sharper double exponential dependence, which is a basis of the observed superselectivity of the TLR9 activation towards the DNA-DNA spacing in the DNA-peptide bundles.

\section{Electrostatic interactions and formation of condensed polyelectrolyte complexes}

Since a number of reviews on electrostatics in soft matter exist in a variety of formats and lengths, we offer only a summary discussion of how DNA forms condensed, orientationallyordered complexes in the presence of cations of various sizes.

DNA and other biological polyelectrolytes (such as F-actin and microtubules) carry uncompensated negative charge. In biologically relevant aqueous conditions, these charges are strongly screened by the dielectric response of water, and by salt solutions. However, this simple picture is incomplete. Counterion entropy can result in surprisingly strong interactions between charged objects in water despite strong screening, via coupling between osmotic and electrostatic interactions. Many experiments have demonstrated that like charged objects repel because of the osmotic pressure of squeezed counterions, and oppositely charged objects repel because of the entropy gain of counterion release. For example, the free energy gain upon binding between two macro-ions scales as $\mathrm{kT}$ multiplied by the number of counterions released (if one neglects ion hydration effects), which can constitute a large thermodynamic driving force.

Poisson-Boltzmann (PB) theory provides quantitative predictions of ion distribution about charged rods ${ }^{35-37}$. In this mean field approximation, properties associated with discrete ions are not considered, including correlations between ions or finite ion size. PB predicts that likecharged objects, such as polyelectrolytes, repel in all salt conditions, including multivalent salts. Increasing the valence of the ions increases the screening contribution and weaken repulsion, but does not invert the sign of the repulsive interaction to attraction.

The above prediction is clearly not in agreement with experiments. Condensation of polyelectrolytes by multivalent ions or/and macroions is widely observed in biological and biomedical contexts. Polyelectrolytes chains such as DNA can 'condense' or collapse into a compact phase from solution as the concentration of oppositely charged multivalent ions or macroions increases. Examples include DNA packing in viruses ${ }^{38,39}$, in bacteria ${ }^{40,41}$, and in many of the DNA complexes that do not lead to TLR9 activation ${ }^{1}$. From a considerable body of theoretical and computational work, we know why highly charged polyelectrolytes can form aggregates. In physical situations with high surface charge densities or with multivalent ions, the organization and dynamics of condensed ions surrounding the polyelectrolyte are important. A series of pioneering Monte Carlo simulations ${ }^{42}$ demonstrated attraction between like-charged DNA. In the last two decades, a large number of theoretical investigations have focused on the physical origins of like-charge attraction ${ }^{43,44}$, and on the changes induced in the organization of 
the polyelectrolyte ${ }^{45,46}$. Correlations between condensed counterions on the polyelectrolyte surface can generate attractions ${ }^{36,42,47,48}$ and organize condensed structures of polyelectrolytes. Oosawa $^{49}$ showed that correlations between thermal fluctuations of the condensed counterion layers can result in attractions. These ideas have been refined by various groups ${ }^{50,51}$. At close distances, local fields due to the spatial arrangement of charges on a macroion can lead to patterns of counterion binding ${ }^{52-54}$. If these ions are ordered, attractions can result as counterions arrange themselves along the surfaces of adjacent macroions in complementary patterns. An elegant picture of interacting Wigner crystals was developed by Rouzina and Bloomfield ${ }^{55}$, Shklovskii ${ }^{56}$, and Lau and Pincus ${ }^{57}$. Recent work in the 'strong coupling' limit ${ }^{58,59}$, where the counterion charge or surface charge density exceed the range of applicability of Poisson-Boltzmann theories ${ }^{60}$ and where correlations between counterions are strong, has predicted the spatial dependence of attractive forces ${ }^{60-62}$. Using a new analytical Wigner-crystal based formulation of strong coupling, Šamaj and Trizac ${ }^{63}$ were able to eliminate divergences from the virial expansion approach, and obtain results on charged plates that agree well with Monte Carlo simulations.

The condensing agent for the DNA polyelectrolyte can be a multivalent macroion like a peptide or protein rather than a simple multivalent cation. Examples include chromosomes ${ }^{64-68}$, and synthetic gene delivery systems based on cationic polymers ${ }^{21,69}$ and dendrimers ${ }^{20,70}$. The behavior of these condensed DNA phases becomes even richer with increasing complexity in the condensing cations. Lipids are amphiphiles that are structurally quite different from facially amphiphilic antimicrobial peptides considered in this review ${ }^{1}$. DNA-cationic lipid complexes have recently received extensive experimental and theoretical scrutiny, since they are empirically known to be efficient gene delivery systems ${ }^{71-80}$. A polymorphism of different selfassembled structures in these DNA-lipid complexes (such as the lamellar, hexagonal, and inverted hexagonal phases) with different transfection efficiencies has been found using synchrotron $x$-ray scattering ${ }^{80-82}$. It is interesting to note that other biological polyelectrolytes can participate in similar forms of electrostatic self-assembly. Cytoskeletal polyelectrolytes include F-actin, intermediate filaments, and microtubules. These polyelectrolytes have different effective diameters, surface charge densities, and flexibilities, and can interact with oppositely charged cations, or cationic proteins, or cationic amphiphilies to generate a diverse range of structures ${ }^{83-}$ ${ }^{88}$. A full description of these structures is however beyond the scope of the present review.

\section{Finite-sized DNA bundles and multivalent binding}

The degree of multivalent binding between TLR9 receptors and the 'grill' of DNA ligands presented by the DNA complex depend in part on the lateral size of the DNA bundle. It is interesting to examine the size of the assembled complexes. Experiments show one generic feature: the electrostatic complexes always have finite size ${ }^{89,90}$. This is puzzling since once an attractive interaction sets in, we expect the polyelectrolyte aggregates to grow to macroscopic sizes and phase separate, until the supply of individual polyelectrolytes are depleted ${ }^{91,92}$. It has been suggested that kinetics may limit the size of these aggregates. In solution, most polyelectrolyte rods in principle meet at an angle with a repulsive interaction, and thereby slow down the growth kinetics, and result in bundles of finite size that are not thermodynamically 
stable $^{93}$. Finite-sized bundles at equilibrium may occur if steric effects from the finite size of counterion prevent the bundle from reaching charge neutrality ${ }^{94}$. Likewise, frustration inherent in the bundle structure may cost an energy penalty ${ }^{94,95}$. Computer simulations also suggest a tendency towards a finite aggregate size ${ }^{96,97}$. Recent theoretical work has proposed that finitesized condensed bundles are a natural consequence of the chirality of the semiflexible polyelectrolytes, which results in elastic strain from polyelectrolyte rod bending and lattice shear increases as the bundle grows ${ }^{98}$.

\section{Outlook}

With a small number of outstanding exceptions, physicists and immunologists do not routinely work together. The goal of this expository review is to familiarize the physicist reader with some of the attending basic immunology and hopefully also the immunologist reader with some of the ideas in polyelectrolyte physics and multivalent binding that are in play within this problem, with the hope that the new perspectives gained can impact the development of fundamental biology and therapeutic strategies. 1

\section{Acknowledgements}

We thank Amber Kaplan for her helpful discussions. This work is supported by NSF grant DMR1411329, EU grants ARG-ERC-COLSTRUCTION 227758 and ITN-COMPLOIDS 234810, by the Herchel Smith fund, and by the Slovenian research agency through Grant P1-0055, and the Swiss National Science Foundation (FN 310030-144072). X-ray research was conducted at Stanford Synchrotron Radiation Lightsource, SLAC National Laboratory, supported by the US DOE Office of Basic Energy Sciences under Contract No. DE-AC02-76SF00515, the Advanced Light Source, supported by the US DOE Office of Basic Energy Sciences under Contract No. DE-AC02-05CH11231, and at the UCLA CNSI. 


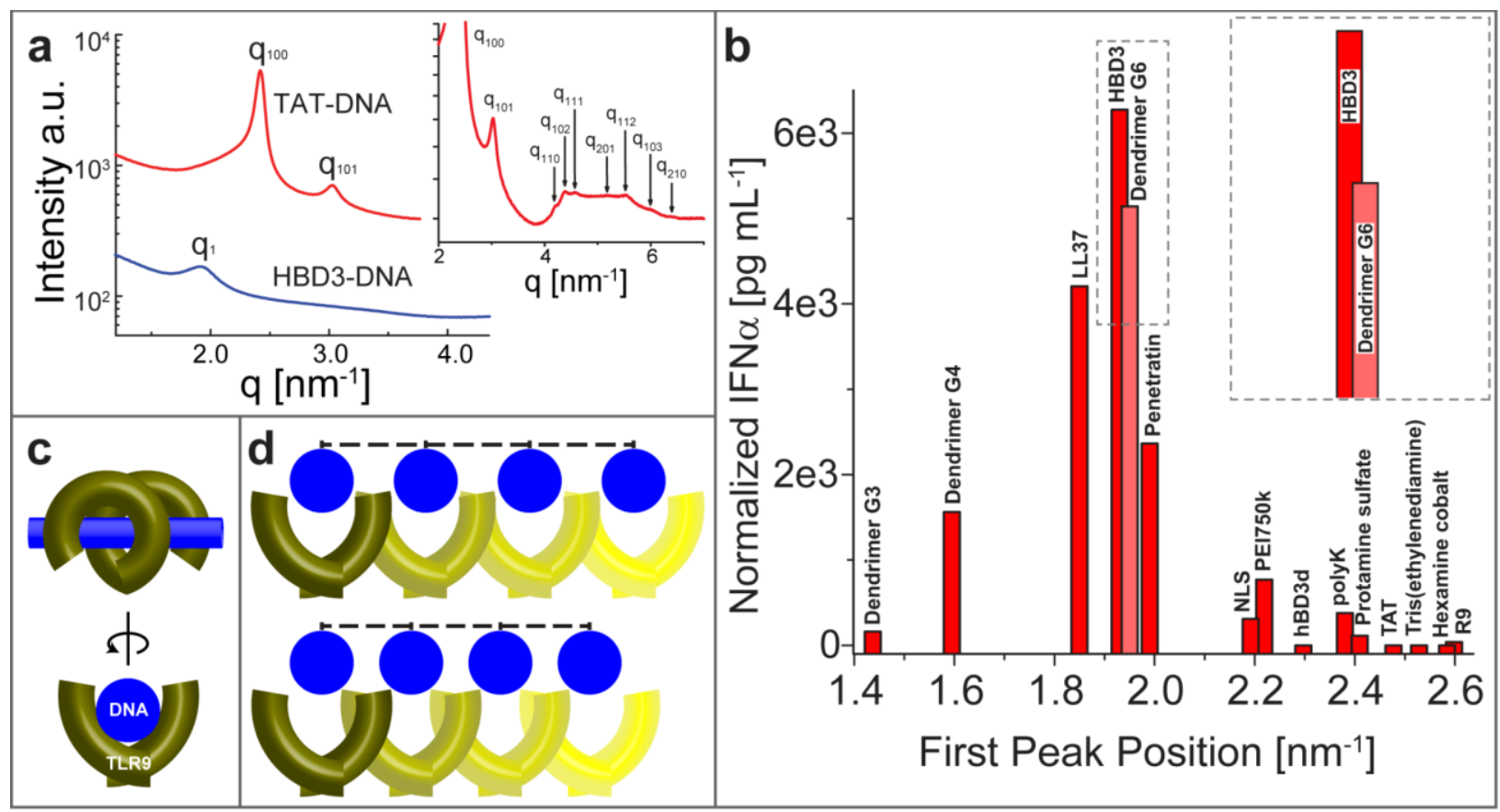

Figure 1: Interferon production in pDCs through TLR9 binding depends on the inter-DNA spacing in DNA complexes. a, SAXS data from DNA-HBD3 complex and from DNA-TAT complex. b, IFN- $\alpha$ production by pDCs stimulated with DNA complexes shows a strong correspondence with SAXS measurements of their first diffraction peak q-positions. A narrow range of inter-DNA spacings result in high IFN- $\alpha$ production levels. $c$, Schematic diagram of the TLR9-dsDNA complex. d, Schematic diagram of how different DNA spacings can impact TLR9 activation, based on how well the DNA 'grill' fits into the arrangement of TLR9 receptors. 
A
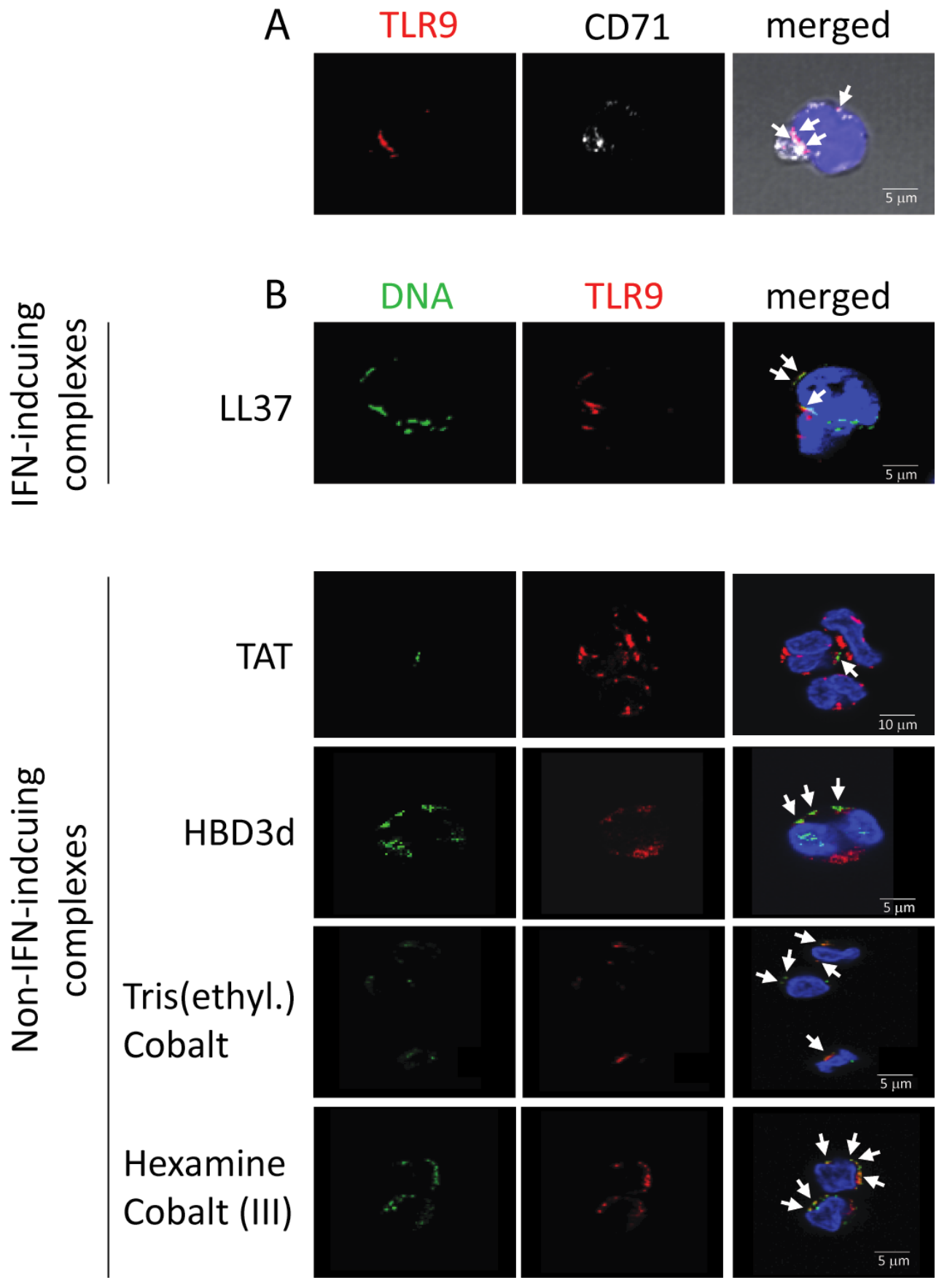

Figure 2: Fluorescence microscopy shows that both IFN inducing DNA complexes and non-IFN inducing DNA complexes have endosomal access. Experimental details are described elsewhere ${ }^{1}$. 

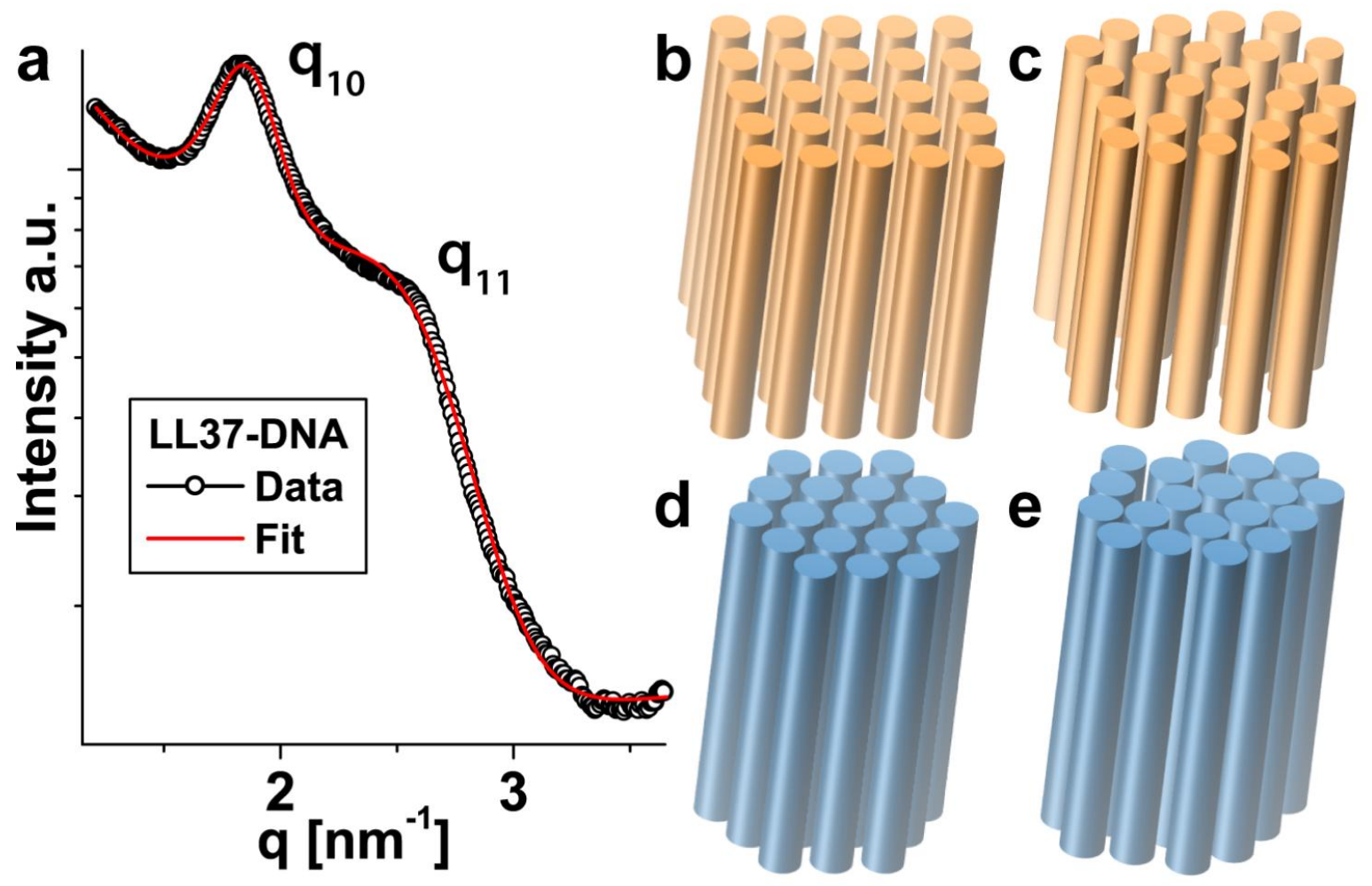

Figure 3: a, SAXS spectra from LL37-DNA complexes. Schematic representations of DNA complexes that induce strong IFN production $(b, c)$, and those that induce weak IFN production $(d, e)$.

\section{References:}

1. Schmidt, N. W. et al. Liquid-crystalline ordering of antimicrobial peptide-DNA complexes controls TLR9 activation. Nat Mater 14, 696-700 (2015).

2. Lande, R. et al. Plasmacytoid dendritic cells sense self-DNA coupled with antimicrobial peptide. 449, 564-569 (2007).

3. Platanias, L. C. Mechanisms of type-I- and type-II-interferon-mediated signalling. Nat Rev Immunol 5, 375-386 (2005).

4. Monroe, K. M., McWhirter, S. M. \& Vance, R. E. Induction of type I interferons by bacteria. Cellular Microbiology 12, 881-890 (2010).

5. Blasius, A. L. \& Beutler, B. Intracellular Toll-like Receptors. Immunity 32, 305-315 (2010).

6. González-Navajas, J. M., Lee, J., David, M. \& Raz, E. Immunomodulatory functions of type I interferons. Nat Rev Immunol 12, 125-135 (2012).

7. Barbalat, R., Ewald, S. E., Mouchess, M. L. \& Barton, G. M. Nucleic acid recognition by the innate immune system. Annu. Rev. Immunol. 29, 185-214 (2011).

8. Haas, T. et al. The DNA sugar backbone 2' deoxyribose determines toll-like receptor 9 activation. Immunity 28, 315-323 (2008).

9. Dorschner, R. A. et al. Cutaneous Injury Induces the Release of Cathelicidin AntiMicrobial Peptides Active Against Group A Streptococcus. Journal of Investigative 
Dermatology 117, 91-97 (2001).

10. Gallo, R. L. et al. Syndecans, cell surface heparan sulfate proteoglycans, are induced by a proline-rich antimicrobial peptide from wounds. 91, 11035-11039 (1994).

11. Liu, L. et al. Structure and mapping of the human $\beta$-defensin HBD-2 gene and its expression at sites of inflammation. Gene 222, 237-244 (1998).

12. Ong, P. Y. et al. Endogenous Antimicrobial Peptides and Skin Infections in Atopic Dermatitis. N Engl J Med 347, 1151-1160 (2002).

13. Kahlenberg, J. M. \& Kaplan, M. J. Little peptide, big effects: the role of LL-37 in inflammation and autoimmune disease. J. Immunol. 191, 4895-4901 (2013).

14. Di Domizio, J. et al. Nucleic acid-containing amyloid fibrils potently induce type I interferon and stimulate systemic autoimmunity. Proc Natl Acad Sci USA 109, 1455014555 (2012).

15. Li, Y., Berke, I. C. \& Modis, Y. DNA binding to proteolytically activated TLR9 is sequenceindependent and enhanced by DNA curvature. EMBO J. 31, 919-931 (2012).

16. Tian, J. et al. Toll-like receptor 9-dependent activation by DNA-containing immune complexes is mediated by HMGB1 and RAGE. Nat Immunol 8, 487-496 (2007).

17. Yanai, $\mathrm{H}$. et al. HMGB proteins function as universal sentinels for nucleic-acid-mediated innate immune responses. 462, 99-103 (2009).

18. Gilliet, M. \& Lande, R. Antimicrobial peptides and self-DNA in autoimmune skin inflammation. Curr. Opin. Immunol. 20, 401-407 (2008).

19. Brooks, H., Lebleu, B. \& Vivès, E. Tat peptide-mediated cellular delivery: back to basics. Advanced Drug Delivery Reviews 57, 559-577 (2005).

20. Evans, H. M. et al. Structural polymorphism of DNA-dendrimer complexes. Phys. Rev. Lett. 91, 075501 (2003).

21. DeRouchey, J., Netz, R. R. \& Radler, J. O. Structural investigations of DNA-polycation complexes. Eur. Phys. J. E 16, 17-28 (2005).

22. Choe, J., Kelker, M. S. \& Wilson, I. A. Crystal structure of human toll-like receptor 3 (TLR3) ectodomain. Science 309, 581-585 (2005).

23. Liu, L. et al. Structural basis of toll-like receptor 3 signaling with double-stranded RNA. Science 320, 379-381 (2008).

24. Kang, J. Y. \& Lee, J.-O. Structural biology of the Toll-like receptor family. Annu. Rev. Biochem. 80, 917-941 (2011).

25. Martinez-Veracoechea, F. J., Martinez-Veracoechea, F. J. \& Frenkel, D. Designing super selectivity in multivalent nano-particle binding. Proc Natl Acad Sci USA 108, 1096310968 (2011).

26. Goodridge, H. S. et al. Activation of the innate immune receptor Dectin-1 upon formation of a /phagocytic synapse/'. 472, 471-475 (2011).

27. Murphy, K. Janeway's Immunobiology. (Garland Pub, 2014).

28. Snapper, C. M., Kehry, M. R., Castle, B. E. \& Mond, J. J. Multivalent, but not divalent, antigen receptor cross-linkers synergize with CD40 ligand for induction of Ig synthesis and class switching in normal murine B cells. A redefinition of the TI-2 vs T celldependent antigen dichotomy. J. Immunol. 154, 1177-1187 (1995).

29. Lesley, J., Hascall, V. C., Tammi, M. \& Hyman, R. Hyaluronan binding by cell surface CD44. Journal of Biological Chemistry 275, 26967-26975 (2000).

30. Puré, E. \& Cuff, C. A. A crucial role for CD44 in inflammation. Trends Mol Med 7, 213221 (2001).

31. Toole, B. P. Hyaluronan-CD44 Interactions in Cancer: Paradoxes and Possibilities. Clin. Cancer Res. 15, 7462-7468 (2009).

32. Weissleder, R., Kelly, K., Sun, E. Y., Shtatland, T. \& Josephson, L. Cell-specific targeting of nanoparticles by multivalent attachment of small molecules. Nat Biotechnol 23, 14181423 (2005). 
33. Dubacheva, G. V., Curk, T., Auzély-Velty, R., Frenkel, D. \& Richter, R. P. Designing multivalent probes for tunable superselective targeting. Proc Natl Acad Sci USA 112, 5579-5584 (2015).

34. Albertazzi, L. et al. Spatiotemporal control and superselectivity in supramolecular polymers using multivalency. Proc Natl Acad Sci USA 110, 12203-12208 (2013).

35. Draper, D. E., Grilley, D. \& Soto, A. M. Ions and RNA folding. Annu Rev Biophys Biomol Struct 34, 221-243 (2005).

36. Anderson, C. F. \& RECORD, M. T. Ion Distributions Around Dna and Other Cylindrical Polyions - Theoretical Descriptions and Physical Implications. Annu Rev Biophys Biophys Chem 19, 423-465 (1990).

37. Sharp, K. A. \& Honig, B. Electrostatic Interactions in Macromolecules - Theory and Applications. Annu Rev Biophys Biophys Chem 19, 301-332 (1990).

38. Odijk, T. Statics and dynamics of condensed DNA within phages and globules. Philos Trans A Math Phys Eng Sci 362, 1497-1517 (2004).

39. Kindt, J., Tzlil, S., Ben-Shaul, A. \& Gelbart, W. M. DNA packaging and ejection forces in bacteriophage. 98, 13671-13674 (2001).

40. Reich, Z., Wachtel, E., Wachtel, E. J. \& Minsky, A. Liquid-crystalline mesophases of plasmid DNA in bacteria. Science 264, 1460-1463 (1994).

41. Englander, J. et al. DNA toroids: framework for DNA repair in Deinococcus radiodurans and in germinating bacterial spores. J. Bacteriol. 186, 5973-5977 (2004).

42. Guldbrand, L., Nilsson, L. G. \& Nordenskiöld, L. A Monte Carlo simulation study of electrostatic forces between hexagonally packed DNA double helices. J. Chem. Phys. 85, 6686-6698 (1986).

43. Barbosa, M. C., Deserno, M. \& Holm, C. A stable local density functional approach to ionion correlations. Europhys. Lett. 52, 80-86 (2000).

44. Ray, J. \& Manning, G. S. An attractive force between two rodlike polyions mediated by the sharing of condensed counterions. Langmuir 10, 2450-2461 (1994).

45. H Schiessel, A., Schiessel, H., P Pincus \& Pincus, P. Counterion-Condensation-Induced Collapse of Highly Charged Polyelectrolytes. Macromolecules 31, 7953-7959 (1998).

46. Solis, F. J. \& la Cruz, de, M. O. Collapse of flexible polyelectrolytes in multivalent salt solutions. J. Chem. Phys. 112, 2030-2035 (2000).

47. Lamm, G., Wong, L. \& Pack, G. R. Monte Carlo and Poisson-Boltzmann calculations of the fraction of counterions bound to DNA. Biopolymers 34, 227-237 (1994).

48. MacKerell, A. D., Jr. \& Nilsson, L. Molecular dynamics simulations of nucleic acid-protein complexes. Current Opinion in Structural Biology 18, 194-199 (2008).

49. Oosawa, F. Interaction between parallel rodlike macroions. Biopolymers 6, 1633-1647 (1968).

50. Golestanian, R., Kardar, M. \& Liverpool, T. B. Collapse of Stiff Polyelectrolytes due to Counterion Fluctuations. Phys. Rev. Lett. 82, 4456-4459 (1999).

51. Ha, B. Y. \& Liu, A. J. Counterion-Mediated Attraction between Two Like-Charged Rods. Phys. Rev. Lett. 79, 1289-1292 (1997).

52. la Cruz, de, M. O. et al. Precipitation of highly charged polyelectrolyte solutions in the presence of multivalent salts. J. Chem. Phys. 103, 5781-5791 (1995).

53. Tan, Z.-J. \& Chen, S.-J. Ion-Mediated Nucleic Acid Helix-Helix Interactions. Biophysj 91, 518-536 (2006).

54. Travesset, A. \& Vaknin, D. Bjerrum pairing correlations at charged interfaces. Europhys. Lett. 74, 181-187 (2006).

55. Rouzina, I. \& Bloomfield, V. A. Macroion Attraction Due to Electrostatic Correlation between Screening Counterions. 1. Mobile Surface-Adsorbed lons and Diffuse lon Cloud. J. Phys. Chem. 100, 9977-9989 (1996).

56. Shklovskii, B. I. Wigner Crystal Model of Counterion Induced Bundle Formation of Rodlike 
Polyelectrolytes. Phys. Rev. Lett. 82, 3268-3271 (1999).

57. Lau, A. W. C., Levine, D. \& Pincus, P. Novel Electrostatic Attraction from Plasmon Fluctuations. Phys. Rev. Lett. 84, 4116-4119 (2000).

58. Moreira, A. G. \& Netz, R. R. Strong-coupling theory for counter-ion distributions. Europhys. Lett. 52, 705-711 (2007).

59. Moreira, A. G. \& Netz, R. R. Binding of Similarly Charged Plates with Counterions Only. Phys. Rev. Lett. 87, 078301 (2001).

60. Naji, A. \& Netz, R. R. Counterions at Charged Cylinders: Criticality and Universality beyond Mean-Field Theory. Phys. Rev. Lett. 95, 185703 (2005).

61. Deserno, M., Arnold, A. \& Holm, C. Attraction and ionic correlations between charged stiff polyelectrolytes. Macromolecules (2003). doi:10.1021/ma020923

62. Naji, A., Jungblut, S., Moreira, A. G. \& Netz, R. R. Electrostatic interactions in strongly coupled soft matter. Physica A: Statistical Mechanics and its Applications 352, 131-170 (2005).

63. Šamaj, L. \& Trizac, E. Wigner-crystal formulation of strong-coupling theory for counterions near planar charged interfaces. Phys. Rev. E 84, 041401 (2011).

64. Mohammad-Rafiee, F. \& Golestanian, R. Elastic Correlations in Nucleosomal DNA Structure. Phys. Rev. Lett. 94, 238102 (2005).

65. Richmond, T. J., Finch, J. T., Rushton, B., Rhodes, D. \& Klug, A. Structure of the nucleosome core particle at $7 \AA$ resolution. 311, 532-537 (1984).

66. Luger, K., Mäder, A. W., Richmond, R. K., Sargent, D. F. \& Richmond, T. J. Crystal structure of the nucleosome core particle at 2.8 A resolution. 389, 251-260 (1997).

67. Schiessel, $H$. The nucleosome: A transparent, slippery, sticky and yet stable DNA-protein complex. Eur. Phys. J. E 19, 251-262 (2006).

68. Chen, Y. et al. Revealing transient structures of nucleosomes as DNA unwinds. Nucleic Acids Research 42, 8767-8776 (2014).

69. Boussif, O. et al. A versatile vector for gene and oligonucleotide transfer into cells in culture and in vivo: polyethylenimine. 92, 7297-7301 (1995).

70. Kukowska-Latallo, J. F. et al. Efficient transfer of genetic material into mammalian cells using Starburst polyamidoamine dendrimers. 93, 4897-4902 (1996).

71. Radler, J. O., Koltover, I., Salditt, T. \& Safinya, C. R. Structure of DNA-cationic liposome complexes: DNA intercalation in multilamellar membranes in distinct interhelical packing regimes. Science 275, 810-814 (1997).

72. Felgner, P. L. et al. Lipofection: a highly efficient, lipid-mediated DNA-transfection procedure. 84, 7413-7417 (1987).

73. Felgner, P. L. Gene therapeutics. Nature 349, 351-352 (1991).

74. Gustafsson, J., Arvidson, G., Karlsson, G. \& Almgren, M. Complexes between cationic liposomes and DNA visualized by cryo-TEM. Biochimica et Biophysica Acta (BBA) Biomembranes 1235, 305-312 (1995).

75. Sternberg, B., Sorgi, F. L. \& Huang, L. New structures in complex formation between DNA and cationic liposomes visualized by freeze-fracture electron microscopy. FEBS LETTERS 356, 361-366 (1994).

76. Danilo D Lasic, Helmut Strey, Mark C A Stuart, Rudolf Podgornik, A. \& Frederik, P. M. The Structure of DNA-Liposome Complexes. J. Am. Chem. Soc. 119, 832-833 (1997).

77. May, S. \& BenShaul, A. DNA-lipid complexes: Stability of honeycomb-like and spaghettilike structures. Biophysj 73, 2427-2440 (1997).

78. Bruinsma, R. Electrostatics of DNA-cationic lipid complexes: isoelectric instability. Eur. Phys. J. B 4, 75-88 (1998).

79. Harries, D., May, S., Gelbart, W. M. \& Ben-Shaul, A. Structure, Stability, and Thermodynamics of Lamellar DNA-Lipid Complexes. Biophysj 75, 159-173 (1998).

80. Koltover, I., Salditt, T., Rädler, J. O. \& Safinya, C. R. An Inverted Hexagonal Phase of 
Cationic Liposome-DNA Complexes Related to DNA Release and Delivery. 281, 78-81 (1998).

81. Safinya, C. R. Structures of lipid-DNA complexes: supramolecular assembly and gene delivery. Current Opinion in Structural Biology (2001).

82. Ewert, K. K., Evans, H. M. \& Zidovska, A. A columnar phase of dendritic lipid-based cationic liposome-DNA complexes for gene delivery: hexagonally ordered cylindrical micelles embedded in a DNA .... Journal of the ... (2006).

83. Wong, G. C. L. et al. Lamellar Phase of Stacked Two-Dimensional Rafts of Actin Filaments. Phys. Rev. Lett. 91, 018103-4 (2003).

84. Wong, G. C. L. Hierarchical Self-Assembly of F-Actin and Cationic Lipid Complexes: Stacked Three-Layer Tubule Networks. Science 288, 2035-2039 (2000).

85. Raviv, U., Needleman, D. J. \& Li, Y. Cationic liposome-microtubule complexes: Pathways to the formation of two-state lipid-protein nanotubes with open or closed ends. in (2005).

86. Needleman, D. J. et al. Higher-order assembly of microtubules by counterions: from hexagonal bundles to living necklaces. Proc Natl Acad Sci USA 101, 16099-16103 (2004).

87. Sanders, L. K. et al. Control of electrostatic interactions between F-actin and genetically modified lysozyme in aqueous media. Proc Natl Acad Sci USA 104, 15994-15999 (2007).

88. Sanders, L. K. et al. Structure and Stability of Self-Assembled Actin-Lysozyme Complexes in Salty Water. Phys. Rev. Lett. 95, 108302-4 (2005).

89. Lai, G. H., Coridan, R., Zribi, O. V., Golestanian, R. \& Wong, G. C. L. Evolution of Growth Modes for Polyelectrolyte Bundles. Phys. Rev. Lett. 98, 187802-4 (2007).

90. Sayar, M. \& Holm, C. Finite-size polyelectrolyte bundles at thermodynamic equilibrium. Europhys. Lett. 77, 16001-6 (2007).

91. Ha, B. Y. \& Liu, A. J. Interfaces in solutions of randomly charged rods. Physica A: Statistical Mechanics and its Applications 259, 235-244 (1998).

92. Huang, C.-I. \& Olvera de la Cruz, M. Polyelectrolytes in Multivalent Salt Solutions: Monomolecular versus Multimolecular Aggregation. Macromolecules 35, 976-986 (2002).

93. Ha, B. Y. \& Liu, A. J. Kinetics of bundle growth in DNA condensation. Europhys. Lett. 46, 624-8 (1999).

94. Henle, M. \& Pincus, P. Equilibrium bundle size of rodlike polyelectrolytes with counterioninduced attractive interactions. Phys. Rev. E 71, 060801-4 (2005).

95. Angelini, T. E., Liang, H., Wriggers, W. \& Wong, G. C. L. Like-charge attraction between polyelectrolytes induced by counterion charge density waves. 100, 8634-8637 (2003).

96. Limbach, H. J., Sayar, M. \& Holm, C. Polyelectrolyte bundles. J. Phys.: Condens. Matter 16, S2135-S2144 (2004).

97. Stevens, M. J. Bundle Binding in Polyelectrolyte Solutions. Phys. Rev. Lett. 82, 1-4 (1998).

98. Grason, G. M. \& Bruinsma, R. F. Chirality and equilibrium biopolymer bundles. Phys. Rev. Lett. 99, 098101 (2007). 
DNA-polycation complexes

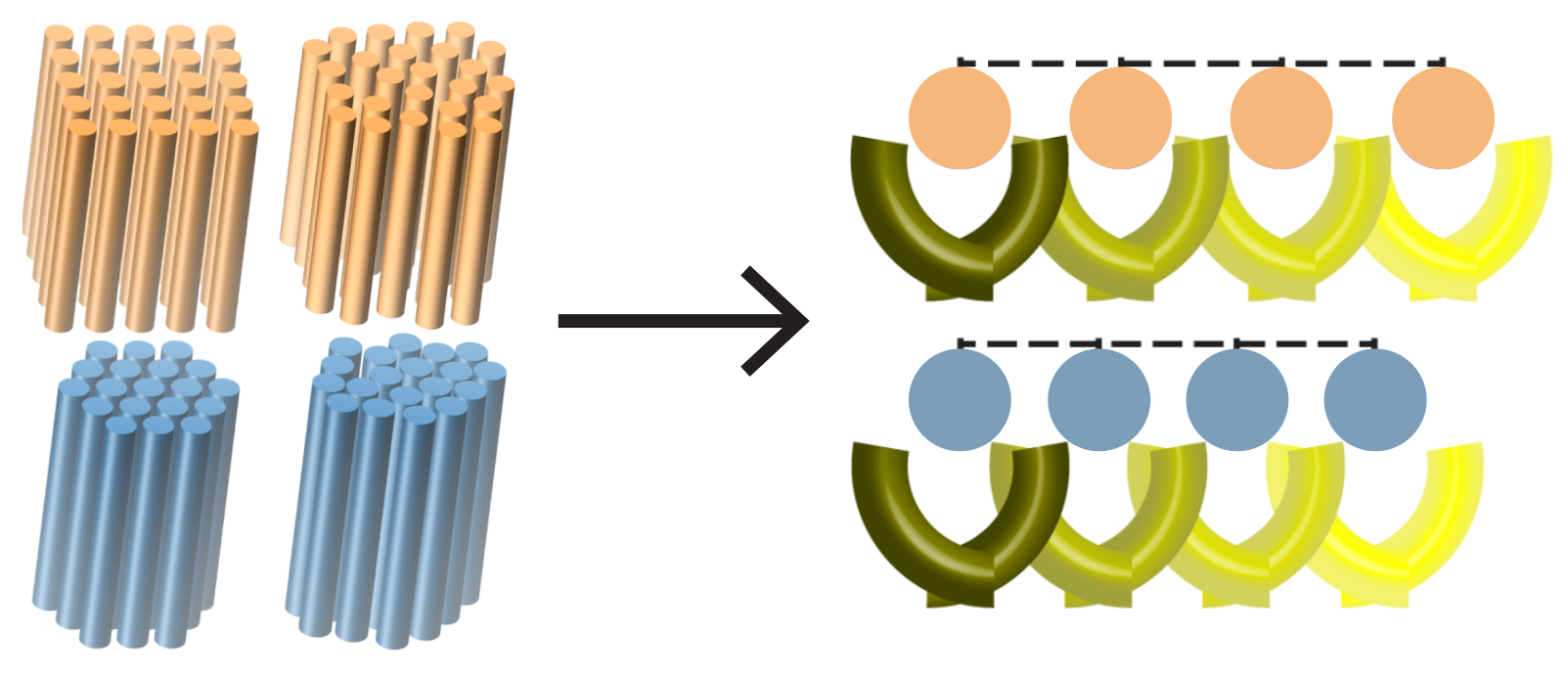

Amplification

No amplification 